\title{
PERFORMANCE OF DIFFERENT ORGANIC FERTILIZERS IN IMPROVING GROWTH AND YIELD OF BORO RICE
}

\author{
T.S. Hoque ${ }^{1}$, I. Jahan ${ }^{1}$, M.R. Islam ${ }^{1 *}$ and M. Ahmed ${ }^{2}$ \\ ${ }^{1}$ Department of Soil Science, Bangladesh Agricultural University, Mymensingh-2202, Bangladesh \\ ${ }^{2}$ Soil Resources Development Institute, Farmgate, Dhaka-1215, Bangladesh
}

\begin{abstract}
A field experiment was conducted at two locations i.e. at Soil Science Field of Bangladesh Agricultural University and at Farmer's field of Fakirakanda village of Mymensingh Sadar to evaluate the effects of different organic fertilizers on the growth and yield of rice (BRRI dhan28). The experiments at each location containing seven treatments were laid out in a randomized complete block design with three replications. The treatments were $\mathrm{T}_{0}$ : Control, $\mathrm{T}_{1}: 75 \%$ RFD; $\mathrm{T}_{2}: 100 \%$ RFD, $\mathrm{T}_{3}: 75 \%$ RFD + Kazi Jaibo Shar $\left(5\right.$ tha $\left.^{-1}\right), T_{4}: 75 \%$ RFD + Kazi Jaibo Shar $\left(3 \mathrm{t} \mathrm{ha}^{-1}\right), \mathrm{T}_{5}$ : $75 \%$ RFD + Poultry manure $\left(3 \mathrm{t} \mathrm{ha}^{-1}\right)$ and $\mathrm{T}_{6}: 75 \%$ RFD + Cow dung $(5 \mathrm{t}$ $\left.\mathrm{ha}^{-1}\right)$. Application of poultry manure as well as Kazi Jaibo Shar showed positive effects on yield attributes, grain and straw yields of rice, nutrient (N, P, K and S) contents and uptake by grain, straw and in total. The performance of $75 \%$ RFD with poultry manure @ $3 \mathrm{t} \mathrm{ha}^{-1}$ was the best in producing yield components, grain and straw yields of rice. At both locations, the performance of same dose $\left(3 \mathrm{t} \mathrm{ha}^{-1}\right)$ of poultry manure and Kazi Jaibo Shar was almost similar in producing growth and yield contributing characters, grain and straw yields, - nutrient content and uptake by rice while each of these manures compensated up to $25 \%$ of recommended chemical fertilizers. Therefore, considering the soil health, poultry manure or Kazi Jaibo Shar @ $3 \mathrm{t} \mathrm{ha}^{-1}$ is recommended for growth and yield enhancement in rice.
\end{abstract}

Keywords: Cow dung, poultry manure, Kazi Jaibo Shar, rice, yield, nutrient uptake

\section{INTRODUCTION}

Bangladesh is predominantly an agrarian country where agriculture sector contributes about 17 percent to the country GDP and employs more than 45 percent of total labour force (BBS, 2016). Rice (Oryza sativa) is the most important cereal crop and

\footnotetext{
* Corresponding author email: mrislam69@yahoo.com
} 
the staple dietary item for the people in Bangladesh and the agriculture sector of the country is largely dominated by rice cultivation. The total area and production of rice are about 11.38 million hectares and 34.71 million metric tons, respectively (BBS, 2016). The average yield of rice in Bangladesh is comparatively lower than those of other South East Asian countries like China, Japan, Korea and Indonesia etc. which might be due to soil fertility depletion as well as poor fertilizer management. Due to ever-rising population, food security has become a key concern in Bangladesh. Consequently, maintenance of soil fertility is necessary for sustainable agriculture and future food security (Majumdar et al., 2016). Hence, to achieve improved and sustainable crop production, the strategy of organic matter and balanced fertilizer management is essential.

Increasing cropping intensity, use of modern varieties (high yielding varieties and hybrids), cultivation of high biomass potential crops, nutrient leaching and unbalanced fertilizer application, with no or little addition of organic manure have resulted in nutrient mining from Bangladesh soils (BARC, 2012). To stop nutrient mining, it is not justified to increase the use of only inorganic fertilizers but the use of organic sources of plant nutrients viz. cow dung, poultry manure, compost, green manure should also be considered. Many farmers use higher amount of inorganic fertilizers while they seldom use organic fertilizers e.g. compost, poultry manure, cow dung. This practice creates imbalance use of fertilizers, which in turn produces a negative impact on crop production. The beneficial aspects of cow dung, poultry manure and compost in increasing crop growth and productivity and maintaining soil fertility have been proven. To increase the efficiency of manure and fertilizer in rice cultivation, it is necessary to identify the suitable combination of manure and fertilizer (Mitu et al. 2017). In recent years, the use of organic fertilizers as alternatives of chemical fertilizers in order to enhance rice production has gained much significance. Kazi Jaibo Shar is a newly developed organic fertilizer based on poultry manure, which is enriched with various nutrient elements. The present study was conducted to clarify the effectiveness of different organic fertilizers at various locations for improving growth, yield and nutritional quality of rice for use as substitute of inorganic fertilizers.

\section{MATERIALS AND METHODS}

A comparative study was performed at two different locations viz. Soil Science Field laboratory of Bangladesh Agricultural University, Mymensingh and a farmer's field at Fakirakanda village of Mymensingh Sadar during the boro season of 2017-2018. The soils of the experimental sites belong to the Sonatala series under the AEZ 9

(Old Brahmaputra Floodplain). The soil of BAU farm was silt loam in texture having 
$\mathrm{pH}$ 6.27, organic matter content $1.95 \%$, total $\mathrm{N} 0.136 \%$, available $\mathrm{P} 3.16 \mathrm{ppm}$, exchangeable K $0.095 \%$ and available $\mathrm{S} 10.5 \mathrm{ppm}$ whereas the soil of farmer's field was loam in texture with $\mathrm{pH} 6.39$, organic matter content $2.5 \%$, total $\mathrm{N} 0.168 \%$, available P 4.76 ppm, exchangeable K 0.13 me\% and available S 12.47 ppm.

. The treatments at both locations were $\mathrm{T}_{0}$ (Control), $\mathrm{T}_{1}(75 \% \mathrm{RFD}), \mathrm{T}_{2}(100 \% \mathrm{RFD})$, $\mathrm{T}_{3}\left(75 \%\right.$ RFD + KaziJaiboShar5 $\left.\mathrm{t} \mathrm{ha}^{-1}\right), \mathrm{T}_{4}\left(75 \%\right.$ RFD + Kazi Jaibo Shar $\left.3 \mathrm{t} \mathrm{ha}^{-1}\right), \mathrm{T}_{5}$ $\left(75 \%\right.$ RFD + Poultry manure $\left.3 \mathrm{t} \mathrm{ha}^{-1}\right)$ and $\mathrm{T}_{6}\left(75 \%\right.$ RFD + Cow dung $\left.5 \mathrm{t} \mathrm{ha}^{-1}\right)$. Experiments was laid out in a Randomized Complete Block Design (RCBD) with three replications and the size of unit plot was $4 \mathrm{~m} \times 3 \mathrm{~m}$. BRRI dhan 28 was used for both of the experiments. Thirty-five days old seedlings of rice were transplanted in the experimental plots on 06 February, 2018 by maintaining a spacing of $20 \mathrm{~cm} \times 20$ $\mathrm{cm}$.

The doses of nitrogen $(\mathrm{N})$, phosphorus $(\mathrm{P})$, potassium $(\mathrm{K})$, sulphur $(\mathrm{S})$ and zinc $(\mathrm{Zn})$ were $150,20,65,18$ and $2 \mathrm{~kg} \mathrm{ha}^{-1}$ following the Fertilizer Recommendation Guide (BARC, 2012) in form of urea, triple super phosphate (TSP), muriate of potash (MoP), gypsum and zinc oxide, respectively. The full doses of chemical fertilizers except urea were applied before transplanting as basal dose to all the experimental plots. Organic manures viz. poultry manure, cow dung and Kazi Jaibo Shar were also applied during final land preparation. Urea was applied in three equal splits as top dressing; first installment at 12 days after transplanting (DAT), second installment at 30 DAT i.e. at maximum tillering stage and third installment at 50 DAT i.e. booting stage of the crop.

Different intercultural operations such as irrigation, weeding, pest control were performed as and when required. The rice was harvested at full maturity on 10 June 2018. The data on growth and yield parameters such as plant height, number of effective tillers hill ${ }^{-1}$, panicle length, number of filled and unfilled grains panicle ${ }^{-1}$, 1000-grain weight, dry and fresh weight of root were recorded at harvest. The grain and straw yields were measured plot wise and expressed as $\mathrm{tha}^{-1}$ on sundry basis.

The collected grain and straw samples from each plot were dried in an oven at $65^{\circ} \mathrm{C}$ for about 48 hours and then ground by a grinding machine. Later, the ground samples were sieved through a 20-mesh sieve. The ground plant materials were stored in paper bags separately and placed in a desiccator. The plant samples were chemically analyzed for determination of $\mathrm{N}, \mathrm{P}, \mathrm{K}$ and $\mathrm{S}$ contents. The total $\mathrm{N}$, available $\mathrm{P}$, exchangeable $\mathrm{K}$ and available $\mathrm{S}$ of plant samples were determined following semimicro Kjeldahl method (Bremner and Mulvaney, 1982), modified Olsen method (Olsen et al., 1954), $\mathrm{NH}_{4} \mathrm{OAc}$ extraction method (Knudsen et al., 1982), and $\mathrm{CaCl}_{2}$ extraction method (Williams and Steinbergs,1959), respectively. 
After chemical analysis of grain and straw samples of rice, the nutrient uptake was calculated from the nutrient content and yield of the crop using the following formula;

$\mathrm{TU}=\frac{\mathrm{NC} \times \mathrm{Y}}{100}$

where, $\mathrm{TU}=$ total nutrient uptake $\left(\mathrm{kg} \mathrm{ha}^{-1}\right), \mathrm{NC}=$ nutrient concentration $(\%)$, and $\mathrm{Y}=$ yield $\left(\mathrm{kg} \mathrm{ha}^{-1}\right)$.

All the data were statistically analyzed by F-test and the mean differences were ranked by Duncan's New Multiple Range Test (DMRT) (Gomez and Gomez, 1984). Differences at $\mathrm{p}<0.05$ were considered significant.

\section{RESULTS AND DISCUSSION}

\section{Growth parameters of BRRI dhan28}

Application of Kazi Jaibo Shar had significant effects on the growth parameters of BRRI dhan28 viz. plant height, root length, fresh and dry weight of root plant ${ }^{-1}$ at both BAU farm as well as in Farmer's field (Table 1). At BAU farm, the highest value for plant height $(99.40 \mathrm{~cm})$ was observed in $\mathrm{T}_{5}$ treatment $(75 \%$ RFD + Poultry manure $3 \mathrm{t} \mathrm{ha}^{-1}$ ) which was statistically similar with those of $\mathrm{T}_{3}$ treatment (75\% RFD + Kazi Jaibo Shar $\left.5 \mathrm{t} \mathrm{ha}^{-1}\right)$ and $\mathrm{T}_{4}\left(75 \%\right.$ RFD + Kazi Jaibo Shar $\left.3 \mathrm{t} \mathrm{ha}^{-1}\right)$ treatments. The shortest plant of $82.33 \mathrm{~cm}$ was found in $\mathrm{T}_{6}$ treatment $(75 \%$ RFD + Cow dung $5 \mathrm{t}$ $\left.\mathrm{ha}^{-1}\right)$. The tallest plant of $83.33 \mathrm{~cm}$ at farmer's field was found in $\mathrm{T}_{2}(100 \% \mathrm{RFD})$ which was statistically identical with those of $T_{3}, T_{4}$ and $T_{5}$. Root length was statistically similar in six treatments but significantly better to control at both locations. For both fresh and dry weight of roots, the highest values (23 and $8.27 \mathrm{~g}$, respectively) were observed in $\mathrm{T}_{4}$ treatment with the lowest values in control (14.33 and $3.87 \mathrm{~g}$, respectively). The growth parameters of BRRI dhan 28 grown at Farmer's field had more or less similar trend (Table 1). The maximum values for root length $(14.27 \mathrm{~cm})$, fresh weight of root $(23.63 \mathrm{~g})$ and dry weight of root $(7.70 \mathrm{~g})$ plant $^{-1}$ were recorded from $\mathrm{T}_{4}$ (Table 1) where Kazi Jaibo Shar was applied @ $3 \mathrm{t} \mathrm{ha}^{-1}$ with 75\% RFD. Tazmin et al. 2015 and Islam et al. 2012 also reported that application of manure and fertilizers enhanced the yield contributing the character of rice. 
Table 1. Effect of different organic fertilizers on growth parameters of BRRI dhan 28

\begin{tabular}{l|c|c|c|c|c|c|c|c}
\hline \multirow{2}{*}{ Treatments } & \multicolumn{5}{|c|}{ BAU farm } & \multicolumn{4}{c}{ Farmer's field } \\
\cline { 2 - 9 } & $\begin{array}{c}\text { Plant } \\
\text { height } \\
(\mathrm{cm})\end{array}$ & $\begin{array}{c}\text { Root } \\
\text { length } \\
(\mathrm{cm})\end{array}$ & $\begin{array}{c}\text { Fresh } \\
\text { weight of } \\
\text { root }(\mathrm{cm})\end{array}$ & $\begin{array}{c}\text { Dry } \\
\text { weight of } \\
\text { root }(\mathrm{cm})\end{array}$ & $\begin{array}{c}\text { Plant } \\
\text { height } \\
(\mathrm{cm})\end{array}$ & $\begin{array}{c}\text { Root } \\
\text { length } \\
(\mathrm{cm})\end{array}$ & $\begin{array}{c}\text { Fresh } \\
\text { weight of } \\
\text { root }(\mathrm{cm})\end{array}$ & $\begin{array}{c}\text { Dry } \\
\text { weight of } \\
\text { root }(\mathrm{cm})\end{array}$ \\
\hline $\mathrm{T}_{0}$ & $83.00 \mathrm{c}$ & $9.63 \mathrm{~b}$ & $14.33 \mathrm{c}$ & $3.87 \mathrm{c}$ & $67.93 \mathrm{bc}$ & $7.70 \mathrm{~b}$ & $15.67 \mathrm{c}$ & $3.20 \mathrm{~b}$ \\
$\mathrm{~T}_{1}$ & $98.40 \mathrm{ab}$ & $13.70 \mathrm{a}$ & $19.60 \mathrm{ab}$ & $6.80 \mathrm{ab}$ & $78.00 \mathrm{ab}$ & $13.20 \mathrm{a}$ & $21.70 \mathrm{a}$ & $6.50 \mathrm{ab}$ \\
$\mathrm{T}_{2}$ & $87.93 \mathrm{bc}$ & $14.07 \mathrm{a}$ & $21.80 \mathrm{a}$ & $6.83 \mathrm{ab}$ & $83.33 \mathrm{a}$ & $12.60 \mathrm{a}$ & $22.83 \mathrm{a}$ & $7.17 \mathrm{a}$ \\
$\mathrm{T}_{3}$ & $90.37 \mathrm{abc}$ & $14.67 \mathrm{a}$ & $19.13 \mathrm{ab}$ & $5.33 \mathrm{bc}$ & $82.73 \mathrm{a}$ & $12.53 \mathrm{a}$ & $22.03 \mathrm{a}$ & $6.60 \mathrm{ab}$ \\
$\mathrm{T}_{4}$ & $96.07 \mathrm{ab}$ & $13.77 \mathrm{a}$ & $23.00 \mathrm{a}$ & $8.27 \mathrm{a}$ & $82.80 \mathrm{a}$ & $14.27 \mathrm{a}$ & $23.63 \mathrm{a}$ & $7.70 \mathrm{a}$ \\
$\mathrm{T}_{5}$ & $99.40 \mathrm{a}$ & $13.97 \mathrm{a}$ & $19.07 \mathrm{~b}$ & $7.53 \mathrm{ab}$ & $80.73 \mathrm{ab}$ & $13.80 \mathrm{a}$ & $23.47 \mathrm{a}$ & $7.23 \mathrm{a}$ \\
$\mathrm{T}_{6}$ & $82.33 \mathrm{c}$ & $13.60 \mathrm{a}$ & $17.90 \mathrm{~b}$ & $4.93 \mathrm{bc}$ & $62.47 \mathrm{c}$ & $12.10 \mathrm{a}$ & $18.90 \mathrm{~b}$ & $3.77 \mathrm{~b}$ \\
$\mathrm{CV}(\%)$ & 6.54 & 11.82 & 18.94 & 22.84 & 9.49 & 15.45 & 6.78 & 27.74 \\
$\mathrm{SE}( \pm)$ & 4.87 & 1.29 & 3.02 & 1.13 & 5.95 & 1.55 & 1.17 & 1.42 \\
\hline
\end{tabular}

$\mathrm{T}_{0:}$ Control; $\mathrm{T}_{1}: 75 \%$ RFD; $\mathrm{T}_{2}: 100 \%$ RFD; $\mathrm{T}_{3}: 75 \%$ RFD + Kazi Jaibo Shar $\left(5\right.$ tha $\left.^{-1}\right) ; \mathrm{T}_{4}: 75 \%$ RFD + Kazi Jaibo Shar $\left(3 \mathrm{t} \mathrm{ha}^{-1}\right) ; \mathrm{T}_{5}: 75 \%$ RFD + Poultry manure $\left(3 \mathrm{tha}^{-1}\right) ; \mathrm{T}_{6}: 75 \%$ RFD + Cow dung (5 tha $\left.{ }^{1}\right) ; \mathrm{CV}=\mathrm{Co}$-efficient of variation; $\mathrm{SE}=$ Standard error of means

\section{Yield parameters of BRRI dhan28}

Number of effective tillers hill ${ }^{-1}$, panicle length, number of filled grains panicle ${ }^{-1}$ and number of unfilled grains panicle ${ }^{-1}$ were significantly affected by different treatments while 1000-grain weight remained statistically unaffected (Table 2). At BAU farm, the treatment $\mathrm{T}_{5}\left(75 \%\right.$ RFD + Poultry manure $\left.3 \mathrm{t} \mathrm{ha}^{-1}\right)$ produced the highest number of effective tillers hill ${ }^{-1}(15.60)$ and the longest panicle $(23.60 \mathrm{~cm})$ which were statistically similar with those of treatments $\mathrm{T}_{1}$ (75\% RFD), $\mathrm{T}_{2}$ (100\% RFD), $\mathrm{T}_{3}(75 \%$ RFD + Kazi Jaibo Shar $\left.5 \mathrm{t} \mathrm{ha}^{-1}\right)$ and $\mathrm{T}_{4}\left(75 \%\right.$ RFD + Kazi Jaibo Shar 3 tha $\left.^{-1}\right)$. Interestingly, the maximum number of filled grains panicle ${ }^{-1}$ (99.47) and the minimum number of unfilled grains panicle ${ }^{-1}(12.27)$ were recorded in $\mathrm{T}_{4}$ treatment. For most of the yield parameters (except 1000-grain weight), the lowest values were observed in control treatment. A little difference was found in the yield contributing characters of rice cultivated at Farmer's field where the highest number of effective tillers hill ${ }^{-1}$ (16.03) was recorded in $\mathrm{T}_{4}$ which has no statistical difference with that of $\mathrm{T}_{5}$. Similar with the result of BAU farm, the tallest panicle of $23.60 \mathrm{~cm}$ and the maximum number of filled grains panicle ${ }^{-1}(96.33)$ were observed in $T_{5}$ which were statistically identical with those of $\mathrm{T}_{2}, \mathrm{~T}_{3}$ and $\mathrm{T}_{4}$ treatments. Notably, the lowest number of effective tillers hill ${ }^{-1}(10.67)$, the lowest panicle length $(18.27 \mathrm{~cm})$ and the lowest number of unfilled grains panicle ${ }^{-1}(8.60)$ were recorded in $\mathrm{T}_{6}$ treatment where cow dung was applied @ $5 \mathrm{t} \mathrm{ha}^{-1}$ with 75\% RFD. Better yield components such as higher panicle length, increased number of effective tillers hill ${ }^{-1}$ and increased number of filled grains panicle ${ }^{-1}$ with poultry manure with inorganic fertilizers were 
also suggested by Islam et al 2018, Ali et al., 2018, Sarker et al., 2015 and Hossaen et al. (2011). Better growth under combination of organic and inorganic fertilizer treatments resulted in higher yield contributing characters that ultimately led to higher grain and straw yields of rice.

Table 2. Effect of different organic fertilizers on yield parameters of BRRI dhan28

\begin{tabular}{|c|c|c|c|c|c|c|c|c|c|c|}
\hline \multirow[b]{2}{*}{ Treatments } & \multicolumn{5}{|c|}{ BAU farm } & \multicolumn{5}{|c|}{ Farmer's field } \\
\hline & $\begin{array}{l}\text { No. of } \\
\text { effective } \\
\text { tillers } \\
\text { hill }^{-1}\end{array}$ & $\begin{array}{l}\text { Panicle } \\
\text { length } \\
(\mathrm{cm})\end{array}$ & $\begin{array}{l}\text { No. of } \\
\text { filled } \\
\text { grains } \\
\text { panicle }^{-1}\end{array}$ & $\begin{array}{l}\text { No. of } \\
\text { unfilled } \\
\text { grains } \\
\text { panicle }^{-1}\end{array}$ & $\begin{array}{c}1000- \\
\text { grain } \\
\text { weight } \\
(\mathrm{g})\end{array}$ & $\begin{array}{c}\text { No. of } \\
\text { effective } \\
\text { tillers } \\
\text { hill }^{-1}\end{array}$ & $\begin{array}{l}\text { Panicle } \\
\text { length } \\
(\mathrm{cm})\end{array}$ & $\begin{array}{l}\text { No. of } \\
\text { filled } \\
\text { grains } \\
\text { panicle }^{-1}\end{array}$ & $\begin{array}{c}\text { No. of } \\
\text { unfilled } \\
\text { grains } \\
\text { panicle }^{-1}\end{array}$ & $\begin{array}{c}1000- \\
\text { grain } \\
\text { weight } \\
(\mathrm{g})\end{array}$ \\
\hline $\mathrm{T}_{0}$ & $8.60 \mathrm{c}$ & $18.40 \mathrm{c}$ & $64.80 \mathrm{c}$ & $17.57 \mathrm{bc}$ & 21.40 & $13.33 b$ & $19.40 \mathrm{~b}$ & $56.20 \mathrm{c}$ & $23.00 \mathrm{a}$ & 22.53 \\
\hline $\mathrm{T}_{1}$ & $14.70 \mathrm{a}$ & $21.60 \mathrm{ab}$ & $95.07 \mathrm{a}$ & $28.13 \mathrm{a}$ & 22.60 & $13.23 b$ & $21.67 \mathrm{a}$ & $77.00 \mathrm{~b}$ & $17.80 \mathrm{ab}$ & 24.37 \\
\hline $\mathrm{T}_{2}$ & $15.33 \mathrm{a}$ & $21.27 \mathrm{ab}$ & $84.07 \mathrm{ab}$ & $28.57 \mathrm{a}$ & 21.80 & $14.33 \mathrm{ab}$ & $22.27 \mathrm{a}$ & $80.93 \mathrm{ab}$ & $15.60 \mathrm{bc}$ & 24.13 \\
\hline $\mathrm{T}_{3}$ & $14.90 \mathrm{a}$ & $22.07 \mathrm{ab}$ & $81.27 \mathrm{~b}$ & $13.80 \mathrm{bc}$ & 24.87 & $13.40 \mathrm{~b}$ & $22.20 \mathrm{a}$ & $81.67 \mathrm{ab}$ & $9.93 \mathrm{c}$ & 22.70 \\
\hline $\mathrm{T}_{4}$ & $14.87 \mathrm{a}$ & $23.33 \mathrm{a}$ & $99.47 \mathrm{a}$ & $12.27 \mathrm{c}$ & 22.07 & $16.03 \mathrm{a}$ & $22.13 a$ & 93.33ab & $13.33 b c$ & 22.73 \\
\hline $\mathrm{T}_{5}$ & $15.60 \mathrm{a}$ & $23.60 \mathrm{a}$ & $92.00 \mathrm{a}$ & $15.13 b c$ & 22.17 & $15.47 \mathrm{a}$ & $22.53 \mathrm{a}$ & $96.33 \mathrm{a}$ & $12.20 \mathrm{bc}$ & 23.97 \\
\hline $\mathrm{T}_{6}$ & $12.30 \mathrm{~b}$ & $19.73 b c$ & $82.00 \mathrm{~b}$ & $23.83 \mathrm{ab}$ & 19.73 & $10.67 \mathrm{c}$ & $18.27 \mathrm{~b}$ & $76.57 \mathrm{~b}$ & $8.60 \mathrm{c}$ & 22.87 \\
\hline $\mathrm{CV}(\%)$ & 8.235 & 6.96 & 15.53 & 27.24 & 8.76 & 7.41 & 5.89 & 12.18 & 26.24 & 3.52 \\
\hline $\mathrm{SE}( \pm)$ & 0.924 & 1.22 & 10.84 & 4.43 & 1.58 & 0.834 & 1.02 & 7.98 & 3.07 & 0.67 \\
\hline
\end{tabular}

$\mathrm{T}_{0:}$ Control; $\mathrm{T}_{1}: 75 \%$ RFD; $\mathrm{T}_{2}: 100 \%$ RFD; $\mathrm{T}_{3}: 75 \%$ RFD + Kazi Jaibo Shar $\left(5 \mathrm{t} \mathrm{ha}^{-1}\right) ; \mathrm{T}_{4}: 75 \%$ RFD + Kazi Jaibo Shar $\left(3 \mathrm{t} \mathrm{ha}^{-1}\right) ; \mathrm{T}_{5}: 75 \%$ RFD + Poultry manure $\left(3 \mathrm{t} \mathrm{ha}^{-1}\right) ; \mathrm{T}_{6}: 75 \%$ RFD + Cowdung $\left(5 \mathrm{tha}^{-1}\right) ; \mathrm{CV}=\mathrm{Co}$-efficient of variation; $\mathrm{SE}=$ Standard error of means

\section{Yield of BRRI dhan28}

Grain yield as well as straw yield of BRRI dhan 28 was significantly influenced with application of Kazi Jaibo Shar (Table 3a). At BAU farm, the grain yield ranged from 3.67 to $6.47 \mathrm{t} \mathrm{ha}^{-1}$ whereas the straw yield ranged from 4.93 to $7.44 \mathrm{t} \mathrm{ha}^{-1}$. For both grain and straw yields, the maximum values were observed in $\mathrm{T}_{5}$ treatment $(75 \%$ RFD + Poultry manure $\left.3 \mathrm{t} \mathrm{ha}^{-1}\right)$ which were statistically similar with those of $\mathrm{T}_{1}(75 \%$ RFD), $\mathrm{T}_{2}\left(100 \%\right.$ RFD), $\mathrm{T}_{3}$ (75\% RFD + Kazi Jaibo Shar $\left.5 \mathrm{t} \mathrm{ha}^{-1}\right)$ and $\mathrm{T}_{4}(75 \%$ RFD + Kazi Jaibo Shar $3 \mathrm{t} \mathrm{ha}^{-1}$ ). The minimum values for both yields were found in control $\left(\mathrm{T}_{0}\right)$. 
Table 3. Effect of different organic fertilizers on grain and straw yields of BRRI dhan 28

\begin{tabular}{c|c|c|c|c}
\hline \multirow{2}{*}{ Treatments } & \multicolumn{2}{|c|}{ BAU farm } & \multicolumn{2}{c}{ Farmer's field } \\
\cline { 2 - 5 } & $\begin{array}{c}\text { Grain yield } \\
\left(\mathrm{t} \mathrm{ha}^{-1}\right)\end{array}$ & $\begin{array}{c}\text { Straw yield } \\
\left(\mathrm{t} \mathrm{ha}^{-1}\right)\end{array}$ & $\begin{array}{c}\text { Grain yield } \\
\left(\mathrm{t} \mathrm{ha}^{-1}\right)\end{array}$ & $\begin{array}{c}\text { Straw yield } \\
\left(\mathrm{t} \mathrm{ha}^{-1}\right)\end{array}$ \\
\hline $\mathrm{T}_{0}$ & $3.67 \mathrm{c}$ & $4.93 \mathrm{c}$ & $3.17 \mathrm{c}$ & $3.80 \mathrm{~d}$ \\
$\mathrm{~T}_{1}$ & $5.97 \mathrm{a}$ & $6.59 \mathrm{ab}$ & $5.33 \mathrm{~b}$ & $6.17 \mathrm{c}$ \\
$\mathrm{T}_{2}$ & $6.07 \mathrm{a}$ & $7.24 \mathrm{a}$ & $5.80 \mathrm{ab}$ & $7.17 \mathrm{ab}$ \\
$\mathrm{T}_{3}$ & $6.06 \mathrm{a}$ & $7.15 \mathrm{a}$ & $5.93 \mathrm{ab}$ & $6.95 \mathrm{bc}$ \\
$\mathrm{T}_{4}$ & $6.17 \mathrm{a}$ & $7.07 \mathrm{a}$ & $5.77 \mathrm{ab}$ & $6.90 \mathrm{bc}$ \\
$\mathrm{T}_{5}$ & $6.47 \mathrm{a}$ & $7.44 \mathrm{a}$ & $6.73 \mathrm{a}$ & $7.89 \mathrm{a}$ \\
$\mathrm{T}_{6}$ & $4.97 \mathrm{~b}$ & $5.87 \mathrm{bc}$ & $5.70 \mathrm{ab}$ & $6.23 \mathrm{c}$ \\
$\mathrm{CV}(\%)$ & 7.86 & 8.10 & 10.40 & 6.83 \\
$\mathrm{SE}( \pm)$ & 0.361 & 0.437 & 0.466 & 0.359 \\
\hline
\end{tabular}

$\mathrm{T}_{0}$ : Control; $\mathrm{T}_{1}: 75 \%$ RFD; $\mathrm{T}_{2}: 100 \%$ RFD; $\mathrm{T}_{3}: 75 \%$ RFD + Kazi Jaibo Shar $\left(5 \mathrm{t} \mathrm{ha}^{-1}\right) ; \mathrm{T}_{4}: 75 \%$ RFD + Kazi Jaibo Shar $\left(3 \mathrm{t} \mathrm{ha}^{-1}\right) ; \mathrm{T}_{5}: 75 \%$ RFD + Poultry manure $\left(3 \mathrm{t} \mathrm{ha}^{-1}\right) ; \mathrm{T}_{6}: 75 \%$ RFD + Cowdung (5 t ha $\left.{ }^{1}\right)$; $\mathrm{CV}=\mathrm{Co}$-efficient of variation; $\mathrm{SE}=$ Standard error of means well as straw yield, different treatments showed their rank in the order of $\mathrm{T}_{5}>\mathrm{T}_{4}>\mathrm{T}_{3}>\mathrm{T}_{2}>\mathrm{T}_{1}>\mathrm{T}_{6}>\mathrm{T}_{0}$.

Almost similar findings were noted in the yield of BRRI dhan 28 grown at Farmer's field where the grain yield varied from 3.17 to $6.73 \mathrm{t} \mathrm{ha}^{-1}$ and the straw yield ranged from 3.80 to $7.89 \mathrm{t} \mathrm{ha}^{-1}$. For both grain and straw yields, treatment $\mathrm{T}_{5}$ produced the highest yields and the control produced the lowest yields. The highest yields from $\mathrm{T}_{5}$ were statistically identical with the yields from $T_{2}, T_{3}, T_{4}$ and $T_{6}$ for grain and with the yield from $T_{2}$ for straw.

At BAU farm, the percent increase in rice yield over control ranged from 35.42 to 76.29 for grain and 19.07 to 50.91 for straw (Fig. 1). On the other hand, the percent increase in rice yield over control at farmer's field varied from 68.14 to 112.30 for grain and 62.37 to 107.63 for straw For both grain and straw yield, $\mathrm{T}_{5}$ treatment gave the highest yield increase over control, respectively. Yield enhancement of rice by applying poultry manure in association with chemical fertilizers was reported by many researchers (Ali et al., 2018; Islam et al., 2012, 2018; Roy et al., 2018; Bhuiyan et al., 2015; Sarker et al., 2015; Tazmin et al. 2015; Issaka et al. 2014; Sangeetha et al., 2013; Hossaen et al., 2011; Hasanuzzaman et al., 2010; Myint et al., 2010). 


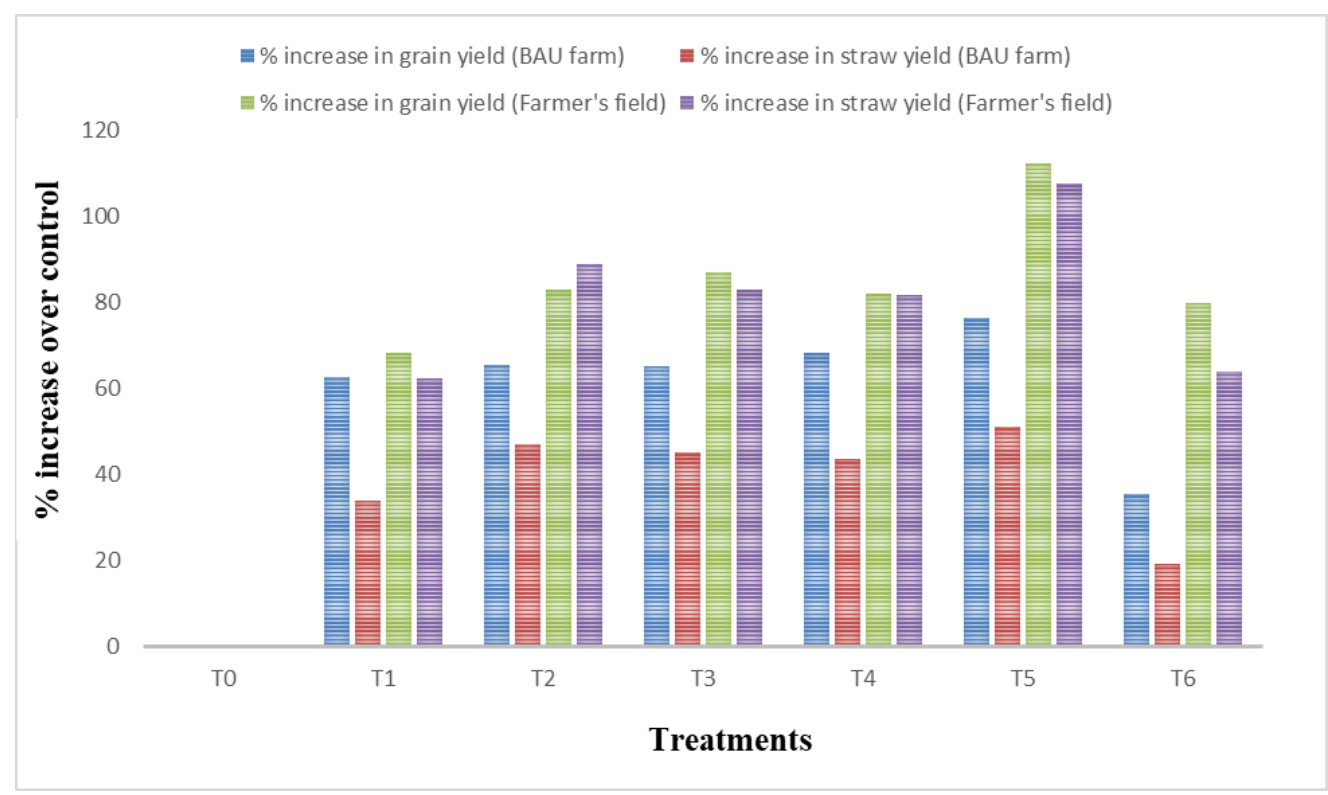

Fig. 1. Percent increase in grain and straw yield of BRRI dhan 28 as influenced by application of different organic fertilizers

\section{Nutrient content of BRRI dhan28}

Application of Kazi Jaibo Shar exerted positive effects on N, P, K and S contents in rice grain and straw at both locations (Table $4 \mathrm{a}$ and $4 \mathrm{~b}$ ). However, a non-significant effect was observed in case of straw $\mathrm{N}$ content at BAU farm and straw $\mathrm{P}$ content at Farmer's field (Table $4 \mathrm{~b}$ ). For BAU farm, the highest $\mathrm{N}$ contents in rice grain and straw $\left(1.21 \%\right.$ and $1.05 \%$, respectively) were recorded in $\mathrm{T}_{5}(75 \%$ RFD + Poultry manure $\left.3 \mathrm{t} \mathrm{ha}^{-1}\right)$. In grain samples, the highest contents of $\mathrm{P}(0.407 \%), \mathrm{K}(0.173 \%)$ and $\mathrm{S}(0.358 \%)$ were found in treatment $\mathrm{T}_{4}\left(75 \% \mathrm{RFD}+\right.$ Kazi Jaibo Shar $\left.3 \mathrm{t} \mathrm{ha}^{-1}\right)$. On the other hand, in straw samples, the maximum contents of $\mathrm{P}(0.151 \%), \mathrm{K}(1.80 \%)$ and $\mathrm{S}(0.302 \%)$ were recorded in $\mathrm{T}_{1}(75 \% \mathrm{RFD}), \mathrm{T}_{5}$ and $\mathrm{T}_{4}(75 \% \mathrm{RFD}+$ Kazi Jaibo Shar $\left.3 \mathrm{tha}^{-1}\right)$, respectively. 
Table 4a. Grain nutrient content of BRRI dhan28 as influenced by different organic fertilizers

\begin{tabular}{c|c|c|c|c|c|c|c|c}
\hline \multirow{2}{*}{ Treatments } & \multicolumn{5}{|c}{ BAU farm } & \multicolumn{4}{c}{ Farmer's field } \\
\cline { 2 - 9 } & $\% \mathrm{~N}$ & $\% \mathrm{P}$ & $\% \mathrm{~K}$ & $\% \mathrm{~S}$ & $\% \mathrm{~N}$ & $\% \mathrm{P}$ & $\% \mathrm{~K}$ & $\% \mathrm{~S}$ \\
\hline $\mathrm{T}_{0}$ & $0.98 \mathrm{c}$ & $0.259 \mathrm{~d}$ & $0.135 \mathrm{~d}$ & $0.271 \mathrm{~b}$ & $0.93 \mathrm{c}$ & $0.246 \mathrm{~d}$ & $0.129 \mathrm{c}$ & $0.295 \mathrm{c}$ \\
$\mathrm{T}_{1}$ & $1.12 \mathrm{~b}$ & $0.301 \mathrm{c}$ & $0.145 \mathrm{~cd}$ & $0.299 \mathrm{~b}$ & $1.12 \mathrm{~b}$ & $0.291 \mathrm{c}$ & $0.144 \mathrm{abc}$ & $0.313 \mathrm{bc}$ \\
$\mathrm{T}_{2}$ & $1.18 \mathrm{ab}$ & $0.291 \mathrm{~cd}$ & $0.143 \mathrm{~cd}$ & $0.307 \mathrm{~b}$ & $1.16 \mathrm{ab}$ & $0.277 \mathrm{c}$ & $0.151 \mathrm{abc}$ & $0.324 \mathrm{~b}$ \\
$\mathrm{~T}_{3}$ & $1.14 \mathrm{~b}$ & $0.335 \mathrm{~b}$ & $0.155 \mathrm{bc}$ & $0.353 \mathrm{a}$ & $1.15 \mathrm{ab}$ & $0.333 \mathrm{~b}$ & $0.161 \mathrm{ab}$ & $0.366 \mathrm{a}$ \\
$\mathrm{T}_{4}$ & $1.16 \mathrm{ab}$ & $0.407 \mathrm{a}$ & $0.173 \mathrm{a}$ & $0.358 \mathrm{a}$ & $1.15 \mathrm{ab}$ & $0.377 \mathrm{a}$ & $0.167 \mathrm{a}$ & $0.368 \mathrm{a}$ \\
$\mathrm{T}_{5}$ & $1.21 \mathrm{a}$ & $0.381 \mathrm{a}$ & $0.168 \mathrm{ab}$ & $0.356 \mathrm{a}$ & $1.20 \mathrm{a}$ & $0.382 \mathrm{a}$ & $0.165 \mathrm{ab}$ & $0.357 \mathrm{a}$ \\
$\mathrm{T}_{6}$ & $1.04 \mathrm{c}$ & $0.281 \mathrm{~cd}$ & $0.143 \mathrm{~cd}$ & $0.279 \mathrm{~b}$ & $0.97 \mathrm{c}$ & $0.273 \mathrm{c}$ & $0.140 \mathrm{bc}$ & $0.289 \mathrm{c}$ \\
$\mathrm{CV}(\%)$ & 3.12 & 5.30 & 6.32 & 6.12 & 3.51 & 4.43 & 8.81 & 4.70 \\
$\mathrm{SE}( \pm)$ & 0.0284 & 0.0139 & 0.0078 & 0.0159 & 0.0315 & 0.0113 & 0.0109 & 0.0127 \\
\hline
\end{tabular}

$\mathrm{T}_{0:}$ Control; $\mathrm{T}_{1}: 75 \%$ RFD; $\mathrm{T}_{2}: 100 \%$ RFD; $\mathrm{T}_{3}: 75 \%$ RFD + Kazi Jaibo Shar $\left(5 \mathrm{t} \mathrm{ha}^{-1}\right) ; \mathrm{T}_{4}: 75 \%$ RFD + Kazi Jaibo Shar $\left(3 \mathrm{t} \mathrm{ha}^{-1}\right) ; \mathrm{T}_{5}: 75 \%$ RFD + Poultry manure $\left(3 \mathrm{t} \mathrm{ha}^{-1}\right) ; \mathrm{T}_{6}: 75 \%$ RFD + Cowdung (5 tha $\left.{ }^{1}\right) ; \mathrm{CV}=\mathrm{Co}$-efficient of variation; $\mathrm{SE}=$ Standard error of means

Similar trend was observed for nutrient contents in grain and straw of BRRI dhan28 cultivated at the Farmer's field. For grain samples, the highest $\mathrm{N}$ and $\mathrm{P}$ contents (1.20\% and $3.82 \%$, respectively) were recorded in $\mathrm{T}_{5}$ treatment while the highest $\mathrm{K}$ and $\mathrm{S}$ contents $\left(0.167 \%\right.$ and $0.368 \%$, respectively) were found in $\mathrm{T}_{4}$ treatment. On the other hand, for straw samples, the highest values for $\mathrm{N}, \mathrm{P}, \mathrm{K}$ and $\mathrm{S}$ contents (1.08\%, $0.144 \%, 1.75 \%$ and $0.291 \%$, respectively) were noted in $\mathrm{T}_{3}, \mathrm{~T}_{2}, \mathrm{~T}_{5}$ and $\mathrm{T}_{4}$, respectively. In general, there was no significant difference between $T_{5}$ and $T_{4}$ for nutrient content in grain and straw samples at both locations. These findings are partially similar to those of Saha et al., 2014; Hossain et al. 2010; Myint et al., 2010 who obtained higher contents of nutrient elements such as N, P, K and S in rice by applying poultry manure with inorganic fertilizers. 
Table 4b. Straw nutrient content of BRRI dhan 28 as influenced by different organic fertilizers

\begin{tabular}{c|c|c|c|c|c|c|c|c}
\hline \multirow{2}{*}{ Treatments } & \multicolumn{5}{|c|}{ BAU farm } & \multicolumn{4}{c}{ Farmer's field } \\
\cline { 2 - 9 } & $\% \mathrm{~N}$ & $\% \mathrm{P}$ & $\% \mathrm{~K}$ & $\% \mathrm{~S}$ & $\% \mathrm{~N}$ & $\% \mathrm{P}$ & $\% \mathrm{~K}$ & $\% \mathrm{~S}$ \\
\hline $\mathrm{T}_{0}$ & 0.96 & $0.121 \mathrm{~cd}$ & $1.43 \mathrm{~d}$ & $0.202 \mathrm{f}$ & $0.91 \mathrm{~b}$ & 0.126 & $1.36 \mathrm{~d}$ & $0.221 \mathrm{e}$ \\
$\mathrm{T}_{1}$ & 0.98 & $0.151 \mathrm{a}$ & $1.48 \mathrm{~cd}$ & $0.250 \mathrm{c}$ & $0.99 \mathrm{ab}$ & 0.143 & $1.47 \mathrm{~cd}$ & $0.257 \mathrm{bcd}$ \\
$\mathrm{T}_{2}$ & 0.99 & $0.142 \mathrm{ab}$ & $1.69 \mathrm{abc}$ & $0.231 \mathrm{~d}$ & $1.00 \mathrm{ab}$ & 0.144 & $1.50 \mathrm{abc}$ & $0.242 \mathrm{cde}$ \\
$\mathrm{T}_{3}$ & 1.02 & $0.126 \mathrm{bcd}$ & $1.65 \mathrm{abcd}$ & $0.251 \mathrm{c}$ & $1.08 \mathrm{a}$ & 0.135 & $1.60 \mathrm{ab}$ & $0.265 \mathrm{abc}$ \\
$\mathrm{T}_{4}$ & 1.01 & $0.122 \mathrm{~cd}$ & $1.79 \mathrm{ab}$ & $0.302 \mathrm{a}$ & $1.02 \mathrm{ab}$ & 0.131 & $1.68 \mathrm{ab}$ & $0.291 \mathrm{a}$ \\
$\mathrm{T}_{5}$ & 1.05 & $0.134 \mathrm{bc}$ & $1.80 \mathrm{a}$ & $0.271 \mathrm{~b}$ & $1.07 \mathrm{a}$ & 0.143 & $1.75 \mathrm{a}$ & $0.281 \mathrm{ab}$ \\
$\mathrm{T}_{6}$ & 0.97 & $0.115 \mathrm{~d}$ & $1.56 \mathrm{bcd}$ & $0.221 \mathrm{e}$ & $0.97 \mathrm{ab}$ & 0.124 & $1.49 \mathrm{abc}$ & $0.235 \mathrm{de}$ \\
$\mathrm{CV}(\%)$ & 4.47 & 6.90 & 7.50 & 2.18 & 5.62 & 10.92 & 9.28 & 6.02 \\
$\mathrm{SE}( \pm)$ & 0.0365 & 0.0073 & 0.0997 & 0.0044 & 0.0461 & 0.012 & 0.1175 & 0.0126 \\
\hline
\end{tabular}

$\mathrm{T}_{0:}$ Control; $\mathrm{T}_{1}: 75 \%$ RFD; $\mathrm{T}_{2}: 100 \%$ RFD; $\mathrm{T}_{3}: 75 \%$ RFD + Kazi Jaibo Shar $\left(5\right.$ tha $\left.^{-1}\right) ; \mathrm{T}_{4}: 75 \%$ RFD + Kazi Jaibo Shar $\left(3 \mathrm{t} \mathrm{ha}^{-1}\right) ; \mathrm{T}_{5}: 75 \%$ RFD + Poultry manure $\left(3 \mathrm{tha}^{-1}\right) ; \mathrm{T}_{6}: 75 \% \mathrm{RFD}+$ Cow dung $\left(5 \mathrm{t} \mathrm{ha}^{-}\right.$ $\left.{ }^{1}\right) ; \mathrm{CV}=\mathrm{Co}$-efficient of variation; $\mathrm{SE}=$ Standard error of means

\section{Nutrient uptake by BRRI dhan28}

Uptake of nutrients (N, P, K and S) by BRRI dhan28 varied significantly due to addition of Kazi Jaibo Shar with inorganic fertilizers (Table 5a and 5b). At BAU farm, the values for grain $\mathrm{N}$-uptake, straw $\mathrm{N}$-uptake and total $\mathrm{N}$-uptake varied from 36.0 to $78.0,47.3$ to 75.7 and 83.7 to $152.3 \mathrm{~kg} \mathrm{ha}^{-1}$, respectively (Table 5a). The maximum values for both grain $\mathrm{N}$-uptake and total $\mathrm{N}$-uptake were found in $\mathrm{T}_{5}$ treatment while the maximum value for straw- $\mathrm{N}$-uptake was observed in $\mathrm{T}_{4}$. Again, the uptake of grain-P, straw $\mathrm{P}$ and total $\mathrm{P}$ ranged from 9.7 to $25.0,6.0$ to 10.3 , and 15.7 to $34.0 \mathrm{~kg} \mathrm{ha}^{-1}$, respectively. In the same manner, $\mathrm{T}_{4}$ and $\mathrm{T}_{5}$ performed the best in grain P uptake and straw P-uptake, respectively; while total P-uptake was same for both treatments. 
Table 5a. Effect of different organic fertilizers on $\mathrm{N}$ and $\mathrm{P}$ uptake by BRRI dhan28

\begin{tabular}{|c|c|c|c|c|c|c|c|c|c|c|c|c|}
\hline \multirow{3}{*}{ Treatments } & \multicolumn{6}{|c|}{ BAU farm } & \multicolumn{6}{|c|}{ Farmer's field } \\
\hline & \multicolumn{3}{|c|}{$\mathrm{N}$ uptake $\left(\mathrm{kg} \mathrm{ha}^{-1}\right)$} & \multicolumn{3}{|c|}{ P uptake $\left(\mathrm{kg} \mathrm{ha}^{-1}\right)$} & \multicolumn{3}{|c|}{$\mathrm{N}$ uptake $\left(\mathrm{kg} \mathrm{ha}^{-1}\right)$} & \multicolumn{3}{|c|}{ P uptake $\left(\mathrm{kg} \mathrm{ha}^{-1}\right)$} \\
\hline & Grain & Straw & Total & Grain & Straw & Total & Grain & Straw & Total & Grain & Straw & Total \\
\hline $\mathrm{T}_{0}$ & $36.0 \mathrm{~d}$ & $47.3 \mathrm{c}$ & $83.7 \mathrm{~d}$ & $9.7 \mathrm{c}$ & $6.0 \mathrm{~b}$ & $15.7 \mathrm{~d}$ & $29.7 \mathrm{c}$ & $35.0 \mathrm{c}$ & $65.0 \mathrm{c}$ & $8.0 \mathrm{~d}$ & $5.0 \mathrm{c}$ & 12.3 \\
\hline $\mathrm{T}_{1}$ & $66.3 b$ & $64.7 \mathrm{ab}$ & $131.0 \mathrm{~b}$ & $18.0 \mathrm{~b}$ & $10.0 \mathrm{a}$ & $27.7 b$ & $60.0 \mathrm{~b}$ & $60.7 b$ & $120.3 b$ & $15.3 \mathrm{c}$ & $9.0 \mathrm{ab}$ & $24.0 \mathrm{~d}$ \\
\hline $\mathrm{T}_{2}$ & 71.3ab & $71.7 \mathrm{a}$ & $143.3 \mathrm{ab}$ & $17.3 b$ & $10.3 \mathrm{a}$ & $28.0 \mathrm{~b}$ & $67.0 \mathrm{~b}$ & $71.7 \mathrm{ab}$ & 139.0ab & $16.0 \mathrm{c}$ & $10.7 \mathrm{a}$ & $26.3 \mathrm{~cd}$ \\
\hline $\mathrm{T}_{3}$ & $68.7 \mathrm{ab}$ & $73.0 \mathrm{a}$ & $142.3 \mathrm{ab}$ & $20.3 b$ & $9.0 \mathrm{a}$ & $29.3 b$ & $68.3 \mathrm{ab}$ & 75.0ab & $143.3 \mathrm{ab}$ & $19.7 b$ & $9.3 \mathrm{ab}$ & $29.3 \mathrm{bc}$ \\
\hline $\mathrm{T}_{4}$ & 71.7ab & $75.7 \mathrm{a}$ & $147.4 \mathrm{ab}$ & $25.0 \mathrm{a}$ & $9.0 \mathrm{a}$ & $34.0 \mathrm{a}$ & $66.3 b$ & $70.0 \mathrm{ab}$ & $136.3 \mathrm{ab}$ & $21.7 b$ & $9.0 \mathrm{ab}$ & $30.7 b$ \\
\hline $\mathrm{T}_{5}$ & $78.0 \mathrm{a}$ & $74.7 \mathrm{a}$ & $152.3 \mathrm{a}$ & $24.7 \mathrm{a}$ & $9.3 \mathrm{a}$ & $34.0 \mathrm{a}$ & $80.7 \mathrm{a}$ & $84.0 \mathrm{a}$ & $164.7 \mathrm{a}$ & $25.7 \mathrm{a}$ & $11.0 \mathrm{a}$ & $37.3 \mathrm{a}$ \\
\hline $\mathrm{T}_{6}$ & $52.0 \mathrm{c}$ & $57.0 \mathrm{bc}$ & $108.7 \mathrm{c}$ & $16.0 \mathrm{bc}$ & $7.0 \mathrm{~b}$ & $23.0 \mathrm{c}$ & $55.3 \mathrm{~b}$ & $66.0 \mathrm{~b}$ & $130.0 \mathrm{~b}$ & $15.7 \mathrm{c}$ & $7.7 b$ & $23.3 \mathrm{~d}$ \\
\hline $\mathrm{CV}(\%)$ & 7.94 & 9.22 & 6.86 & 12.81 & 8.96 & 8.20 & 11.48 & 11.35 & 11.57 & 10.83 & 14.83 & 8.81 \\
\hline $\mathrm{SE}( \pm)$ & 0.0041 & 0.005 & 0.0073 & 0.002 & 0.0006 & 0.0018 & 0.0057 & 0.0061 & 0.0121 & 0.0015 & 0.0011 & 0.0019 \\
\hline
\end{tabular}

$\mathrm{T}_{0:}$ Control; $\mathrm{T}_{1}: 75 \%$ RFD; $\mathrm{T}_{2}: 100 \%$ RFD; $\mathrm{T}_{3}: 75 \%$ RFD + Kazi Jaibo Shar $\left(5\right.$ tha $\left.^{-1}\right) ; \mathrm{T}_{4}: 75 \%$ RFD + Kazi Jaibo Shar $\left(3 \mathrm{t} \mathrm{ha}^{-1}\right) ; \mathrm{T}_{5}: 75 \%$ RFD + Poultry manure $\left(3\right.$ tha $\left.^{-1}\right) ; \mathrm{T}_{6}: 75 \%$ RFD + Cowdung $\left(5\right.$ tha $\left.^{-1}\right)$; $\mathrm{CV}=\mathrm{Co}$-efficient of variation; $\mathrm{SE}=$ Standard error of means

At farmer's field, the values for grain-N uptake, straw $\mathrm{N}$-uptake and total $\mathrm{N}$ uptake ranged from 29.7 to $80.7,35.0$ to 84.0 and 65.0 to $164.7 \mathrm{~kg} \mathrm{ha}^{-1}$, respectively (Table 5a). The highest values for grain-N uptake, straw $\mathrm{N}$-uptake and total $\mathrm{N}$ uptake were found in $\mathrm{T}_{5}$ treatment. Furthermore, the uptake of grain-P, straw $\mathrm{P}$ and total $\mathrm{P}$ varied from 8.0 to $25.7,5.0$ to 11.0 , and 12.3 to $37.3 \mathrm{~kg} \mathrm{ha}^{-1}$, respectively. Similarly, in all three cases of $\mathrm{P}$-uptake (grain $\mathrm{P}$, straw $\mathrm{P}$ and total $\mathrm{P}$ ), the highest values were observed in $\mathrm{T}_{5}$ treatment. While, there was no significant variation in $\mathrm{N}$ and $\mathrm{P}$ uptake between treatment $T_{4}$ and $T_{5}$ for both grain and straw.

At BAU farm, the values for grain-K uptake, straw $\mathrm{K}$-uptake and total $\mathrm{K}$ uptake varied from 4.7 to $10.7,71.0$ to 133.0 and 75.7 to $143.3 \mathrm{~kg} \mathrm{ha}^{-1}$, respectively (Table $5 \mathrm{~b})$. The maximum values for both straw-K uptake and total $\mathrm{K}$ uptake were found in $\mathrm{T}_{4}$ while the maximum value for grain-K uptake was observed in $\mathrm{T}_{5}$ treatment. Again, the uptake of grain-S, straw-S and total S ranged from 10.0 to 23.0, 10.0 to 22.7, and 19.7 to $44.3 \mathrm{~kg} \mathrm{ha}^{-1}$, respectively. In the same manner with $\mathrm{K}$-uptake, treatment $\mathrm{T}_{4}$ caused the highest straw-S uptake and total $\mathrm{S}$ uptake while $\mathrm{T}_{4}$ performed the best in grain S-uptake.

In case of Farmer's field, the values for grain-K uptake, straw K-uptake and total $\mathrm{K}$ uptake varied from 4.0 to $11.0,51.7$ to 138.0 and 56.0 to $149.0 \mathrm{~kg} \mathrm{ha}^{-1}$, respectively while the uptake of grain-S, straw-S and total S ranged from 9.3 to $24.3,8.3$ to 22.0, 
and 17.0 to $46.3 \mathrm{~kg} \mathrm{ha}^{-1}$, respectively (Table $5 \mathrm{~b}$ ). For both $\mathrm{K}$ and $\mathrm{S}$, the maximum values for grain uptake, straw uptake and total uptake were observed in $\mathrm{T}_{5}$ treatment. Notably, there was no statistical difference between treatment $\mathrm{T}_{4}$ and $\mathrm{T}_{5}$ for grain $\mathrm{K}$ uptake as well as S-uptake by grain and straw. In case of all four nutrient elements $(\mathrm{N}, \mathrm{P}, \mathrm{K}$ and $\mathrm{S})$, the lowest uptake was recorded in control treatments at both BAU farm and Farmer's field. The results of our study were partially accorded to those of several comparable studies of some researchers (Roy et al., 2018; Saha et al., 2014; Hossain et al., 2010) who observed increased uptake of N, P, K and S in rice due to application of poultry manure combined with inorganic fertilizers.

\section{CONCLUSION}

From the above results, it may be concluded that organic fertilizers - in form of poultry manure has a potential to increase the growth parameters, yield components, grain and straw yields and nutritional quality of rice. The use of Kazi Jaibo Shar as an organic fertilizer for rice also had positive effects on growth, yield as well as nutrient content and uptake by the crop. The performance of Kazi compost @ $3 \mathrm{t} \mathrm{ha}^{-1}$ is comparable with poultry manure of the same dose in association with chemical fertilizers, and the effect of each of these manures can compensate up to $25 \%$ reduction of recommended chemical fertilizers. Further long-term experimentation in different locations with different crop species is necessary to draw a valid conclusion on effectiveness of these manures.

\section{ACKNOWLEDGEMENT}

The authors are grateful to KAZI FARMS GROUP for a partial financial support for conducting this research work. The research facilities provided by the Department of Soil Science, Bangladesh Agricultural University is also thankfully acknowledged.

\section{REFERENCES}

Ali, M.I., Sarkar, M.A.R., and Paul, S.K. (2018). Influence of plant nutrient management on the yield performance of transplant aman rice (Oryza sativa L.). Archives of Agriculture and Environmental Science, 3(1), 49-53.

BARCl. (2012). Fertilizer Recommendation Guide-2012. BARC, Farmgate, Dhaka.

BBS. (2016). Yearbook of Agricultural Statistics-2016. Statistics and Informatics Division, Ministry of Planning, Govt. People's Republic of Bangladesh.

Bhuiyan, M.K.A., Kamal, A.K.I., Ahmed, F., and Uddin, M.K. (2015). Effectiveness of poultry litter as fertilizer for rice cultivation: prospect of organic fertilizer in Bangladesh. Journal of Biodiversity and Environmental Sciences, 6(3), 8-15. 
Bremner, J.M., and Mulvaney, C.S. (1982). Nitrogen- total. In Methods of Soil Analysis Part 2 edited by Page, Miller and Kenly. American Society of Agronomy, Inc. Publisher, USA, pp. 595-624.

Gomez, K.A., and Gomez, A.A. (1984). Statistical Procedures for Agricultural Research. John Wiley and Sons, New York.

Hasanuzzaman, M., Ahamed, K.U., Rahmatullah, N.M., Akhter, N., Nahar, K., and Rahman, M.L. (2010). Plant growth characters and productivity of wetland rice (Oryza sativa L.) as affected by application of different manures. Emirates Journal of Food and Agriculture, 22(1), 46-58.

Hossaen, M.A., Shamsuddoha, A.T.M., Paul, A.K., Bhuiyan, M.S.I., and Zobaer, A.S.M. (2011). Efficacy of different organic manures and inorganic fertilizer on the yield and yield attributes of Boro rice. The Agriculturists, 9(1 \& 2), 117-125.

Hossain, A.T.M.S., Rahman, F., Saha, P.K., and Solaiman, A.R.M. (2010). Effects of different aged poultry litter on the yield and nutrient balance in Boro rice cultivation. Bangladesh Journal of Agricultural Research, 35(3), 497-505.

Islam, M.S., Sarkar, M.A.R., Uddin, S., and Parvin S. (2012). Yield of fine rice varieties as influenced by integrated management of poultry manure, urea super granules and prilledurea. Journal of Environmental Science and Natural Resources, 5(1), 129-132.

Islam, S., Islam, M.R., and Islam, M.R. (2018). Effects of prilledurea and urea super granule with poultry manure on rice field water property, growth and yield of BRRI Dhan 49 . International Journal of Plant Biology and Research, 6(1), 1080.

Issaka, R.N., Buri, M.M., Nakamura, S., and Tobita, S. (2014). Comparison of different fertilizer management practices on rice growth and yield in the Ashanti region of Ghana.Agriculture, Forestry and Fisheries, 3(5), 374-379.

Knudsen, D. Peterson, G.A., and Pratt, P.F. (1982). Lithium, Sodium and Potassium. In methods of Soil Analysis Prt 2 edited by Page, Miller and Keenly. American Society of Agronomy, Inc. Publisher, USA, pp. 225-245.

Majumdar, K., Sanyal, S.K., Dutta, S.K., Satyanarayana, T., and Singh, V.K. (2016). Nutrient Mining: Addressing the Challenges to Soil Resources and Food Security. In: U. Singh, C. Praharaj, S. Singh, N. Singh (ed.), Biofortification ofFood Crops. Springer, New Delhi. p. 177-198.

Mitu A.S., Khan, M.A., and Rashed, M.R.U. (2017). Effect of fertilizer manure and lime on growth and yield of boro rice in acidic red soil. Agricultural Research and Technology: Open access Journal, 5(5), 555675.

Myint, A.K., Yamakawa, T., Kajihara, Y., and Zenmyo, T. (2010). Application of different organic and mineral fertilizers on the growth, yield and nutrient accumulation of rice in a Japanese ordinary paddy field. Science World Journal, 5(2), 47-54.

Olsen, S.R., Cole, C.U., Watanabe, F.S., and Dean. L.A. (1954). Estimation of available P in soil extraction with sodium bicarbonate. Circular, U.S. Department of Agriculture, 1954, p. 929. 
Roy, S., Kashem, M.A., and Osman, K.T. (20180. The uptake of phosphorous and potassium of rice as affected by different water and organic manure management. Journal of Plant Sciences, 6(2), 31-40.

Saha, R., Saieed, M.A.U., Chowdhury, M.A.K., and Chowdhury, M.A.H. (2014). Influence of humic acid and poultry manure on nutrient content and their uptake by T. aman rice. Journal of the Bangladesh Agricultural University 12(1), 19-24.

Sangeetha, S.P., Balakrishnan, A., and. Devasenapathy, P. (2013). Influence of organic manures on yield and quality of rice (Oryza sativa L.) and blackgram (Vignamungo L.) in rice-blackgramcropping sequence. American Journal of Plant Sciences, 4(5), 11511157.

Sarker, D., Mazumder, S., Kundu, S., Akter, F., and Paul, S.K. (2015). Effect of poultry manure incorporated with nitrogenous and sulphur fertilizer on the growth, yield, chlorophyll and nutrient contents of rice var. BRRI dhan33. Bangladesh Agronomy Journal, 18(1), 99-111.

Tazmin, M.F., Sarkar, M.A.R., and Abuyusuf, M. (2015). Combined level of poultry manure and NPKS fertilizers on growth and yield of Bororice cv. BRRI dhan28 and BRRI dhan29. European Academic Research, 3(5), 5909-5939.

Williams, C.H., and Steinbergs, A. (1959). Soil sulphur fractions as chemical indices of available sulphur in some Australian soils. Australian Journal of Agricultural Research, 10, 340-352. 